\title{
Selective Vulnerability of Dentate Hilar Neurons following Traumatic Brain Injury: A Potential Mechanistic Link between Head Trauma and Disorders of the Hippocampus
}

\author{
Daniel H. Lowenstein, ${ }^{1}$ Mark J. Thomas, ${ }^{2}$ Douglas H. Smith, ${ }^{2}$ and Tracy K. McIntosh² \\ 'Epilepsy Research Laboratory, Department of Neurology, University of California at San Francisco, San Francisco, \\ California 94143 and ${ }^{2}$ Department of Neurosurgery, University of Pennsylvania, 3400 Spruce Street, Philadelphia, \\ Pennsylvania 19104
}

Despite intensive study, the neurobiological basis of epilepsy and persistent memory impairment following traumatic head injury remains unknown. Since abnormalities of the hippocampus are known to be associated with temporal lobe seizures and memory dysfunction, we investigated the effects of experimental traumatic brain injury on hippocampal structure and function in the rat. Using a model of fluidpercussion injury, we have discovered that neurons of the dentate hilus are vulnerable to a brief, unilateral impact to the extradural surface of the brain. One week after trauma, there was a dramatic reduction in hilar neurons ipsilateral to the impact, and a milder but significant decrease in neurons on the contralateral side as well. This neuronal loss was highly selective since adjacent dentate granule and pyramidal neurons appeared relatively unaffected. Immunocytochemistry showed that the hilar cell loss included a loss of somatostatin-immunoreactive neurons, and degeneration stains provided evidence that irreversible hilar injury occurred within $4 \mathrm{hr}$ of impact. To assess the functional effects of the hilar damage, dentate granule cell field potentials were measured in response to perforant path stimulation. This revealed abnormal dentate granule cell hyperexcitability at $2.0 \mathrm{~Hz}$ stimulation in many of the injured animals. The presence of abnormal hyperexcitability correlated with the loss of hilar neurons. Thus, a momentary impact to the surface of the brain can cause selective, bilateral hippocampal injury with associated abnormalities in dentate gyrus physiology. Furthermore, the pattern of cell loss is similar to that observed in some patients with temporal lobe epilepsy. These findings provide a potential mechanistic link between human head trauma and the subsequent development of disorders of the hippocampus such as epilepsy and memory loss.

The neurologic sequelae of head injury range from chronic headaches and memory loss to seizures, gross cognitive dysfunction, and coma (Strauss and Savitsky, 1934; Povlishock and Becker,

\footnotetext{
Received Jan. 7, 1992; revised May 28, 1992; accepted July 7, 1992.

We thank T. McCabe for technical assistance, and L. Jan, M. Miles, D. MochlyRosen, R. Nicoll, H. Scharfman, and R. Sloviter for critical review of the manuscript. This work was supported by NIH Grants NS01424 (D.H.L.) and NS26818 (T.K.M.), VA Merit Review Grant 74R (T.K.M.), and funding from the American Epilepsy Society (D.H.L.) and the Brain Trauma Foundation (T.K.M.).

Correspondence should be addressed to Dr. Daniel H. Lowenstein, Department of Neurology, 4M62, San Francisco General Hospital, 1001 Potrero Avenue, San Francisco, CA 94110.

Copyright (C) 1992 Society for Neuroscience $0270-6474 / 92 / 124846-08 \$ 05.00 / 0$
}

1985). The most extreme disabilities result from severe head trauma, in which there is a direct disruption of brain tissue. However, many patients disabled by head injury suffer milder forms of trauma in which significant brain damage is not evident (Rimel et al., 1981; Kraus, 1987; Levin, 1989). Seizures may occur during the immediate recovery period, and some patients develop chronic, recurrent seizures. For example, there is an association between head trauma and the development of a seizure focus in the temporal lobe (i.e., temporal lobe epilepsy) (Jennett, 1975). Another disability, the "post-concussion syndrome," is characterized by headache, poor memory, and disturbances in attention and concentration (Strauss and Savitsky, 1934; Binder, 1986). Although most patients recover within weeks, some have such severe and protracted impairments in memory and other aspects of cognition that they require months to years to regain normal function (Gentilini et al., 1985; Levin et al., 1987).

To date, there is no cogent explanation for the way in which the relatively nonspecific forces of closed head trauma can lead to the development of focal seizure disorders such as temporal lobe epilepsy. Similarly, it is unclear why head trauma has such a propensity to disturb memory function. Most prior reports have suggested that these CNS disturbances are a consequence of diffuse axonal damage (Strich, 1956; Oppenheimer, 1968; Adams et al., 1982; Pilz, 1983; Povlishock et al., 1983), vascular abnormalities (Lewelt et al., 1980; Dewitt et al., 1986; Yamakami and McIntosh, 1989), or changes in neurochemical transmission (Hayes et al., 1989; Lyeth et al., 1990), but no precise anatomic and functional explanation exists.

Since the hippocampus is believed to have an important role in temporal lobe epilepsy (Engel, 1989) and certain aspects of memory (Amaral, 1987; Squire and Zola-Morgan, 1991), we hypothesized that traumatic brain injury can lead to specific abnormalities of hippocampal structure and function. We addressed this hypothesis using a model of percussion injury in the rat that reproduces many features of head injury in humans (McIntosh et al., 1989). The first question of this study was whether traumatic brain injury causes any specific anatomical changes in the hippocampus. In pilot experiments, inspection of hippocampal sections 1 week after trauma supported this idea, since there was a selective, bilateral loss of neurons in the dentate hilus that increased in severity as the intensity of the impacts increased. Selective loss of hilar neurons following other forms of hippocampal injury such as prolonged electrical stimulation (Sloviter, 1987) and kainic acid-induced excitotoxicity 
(Sloviter and Damiano, 1981) has been associated with the development of abnormalities in dentate granule cell physiology. Therefore, electrophysiological methods were used to address the second main question of this study: does the loss of hilar neurons following traumatic brain injury have any functional consequences in terms of dentate granule cell excitability?

\section{Materials and Methods}

Fluid-percussion injury. The protocols used in these studies were approved by the Animal Research Committees at both the University of California at San Francisco and the University of Connecticut Health Center, and were in accordance with guidelines set by the National Institutes of Health for the humane treatment of animals. Traumatic brain injury was produced by a fluid-percussion device consisting of a Plexiglas cylindrical reservoir, $60 \mathrm{~cm}$ long and $4.5 \mathrm{~cm}$ in diameter, bounded at one end by a cork-covered Plexiglas piston mounted on $\mathrm{O}$ rings. In most experiments, adult Sprague-Dawley rats (350-450 gm) were anesthetized with a single dose of pentobarbital $(60 \mathrm{mg} / \mathrm{kg})$, which was sufficient to maintain surgical anesthesia until 15-30 min after the head impact. To compare potential effects of different anesthetics on patterns of injury, a separate group of animals was first anesthetized with $3 \%$ isoflurane, intubated, and then maintained on $1.5 \%$ isoflurane until 15 min after impact. All animals had a catheter placed in the femoral artery for monitoring of arterial blood pressure and blood gases. The animals were then connected to the fluid-percussion device via a plastic Leur-Loc fitting implanted through a $2.0 \mathrm{~mm}$ craniotomy centered over the left parietal cortex. The entire system was filled with $37^{\circ} \mathrm{C}$ isotonic saline and a metal pendulum was released to strike the piston from a predetermined height. This produced a brief ( $21-23 \mathrm{msec})$ impact against the exposed dura and underlying neural tissue, and directed at the lateral cerebral convexity, midway between lambda and bregma Pressure pulses were measured extracranially by a transducer and recorded on a storage oscilloscope. In experiments done under pentobarbital anesthesia, the force of impact was adjusted to one of three levels: $0.5-1.2$ atmospheres (atm), 1.5-1.6 atm, and 2.0-2.2 atm. Animals anesthetized with isoflurane were exposed to an intermediate impact of 1.8 atm. "Sham" controls had identical anesthetic and surgical preparation without the impact.

Tissue fixation and histologic stains. At different time intervals, animals were anesthetized with pentobarbital and perfused transcardially with saline for $3 \mathrm{~min}$ followed by $4 \%$ paraformaldehyde in $0.1 \mathrm{M}$ PBS (pH 7.4) for $10 \mathrm{~min}$. Brains were postfixed overnight and cut on a vibratome. Sections of 20 and $30 \mu \mathrm{m}$ were mounted directly on gelatincoated slides, air dried, dehydrated, and then rehydrated with graded ethanols and xylene, stained with a toluidine blue stain, dehydrated again with graded ethanols and xylene, and then coverslipped with Permount. The silver impregnation method of Nadler and Evenson (1983) was used with $40 \mu \mathrm{m}$ sections to stain degenerating cells.

Immunocytochemistry. Tissue sections of $50 \mu \mathrm{m}$ were washed in 0.1 M Tris buffer three times for $5 \mathrm{~min}$ each, $1.0 \%$ hydrogen peroxide in Tris buffer for $30 \mathrm{~min}$, Tris buffer for $5 \mathrm{~min}$, Tris A $(0.1 \%$ Triton $\mathrm{X}$ in Tris buffer) for $15 \mathrm{~min}$, Tris $\mathrm{B}(0.1 \%$ Iriton $X$ and $0.005 \% \mathrm{BSA}$ in Tris buffer) for $15 \mathrm{~min}, 10 \%$ normal goat serum in Tris B for $1 \mathrm{hr}$, Tris $A$ for $15 \mathrm{~min}$ and Tris B for $15 \mathrm{~min}$, and then incubated overnight in a polyclonal antibody against somatostatin-14 (Sagar et al., 1985) diluted 1:2000 in Tris B. The following day, the sections were washed in Tris $A$ for $15 \mathrm{~min}$ and Tris $B$ for $15 \mathrm{~min}$, incubated in biotinylated goat anti-rabbit IgG (Vector Labs, Burlingame, CA) at 1:200 dilution in Tris $B$ for $45 \mathrm{~min}$, washed in Tris A for $15 \mathrm{~min}$ and Tris B for $15 \mathrm{~min}$, incubated in avidin-biotin-peroxidase (ABC kit from Vector Labs) at 1:1000 dilution in Tris B for $1 \mathrm{hr}$, washed in Tris buffer three times for $5 \mathrm{~min}$, and then placed into a mixture of $0.5 \mathrm{mg} / \mathrm{ml}$ diaminobenzidine tetrahydrochloride, $3 \mathrm{mg} / \mathrm{ml}$ glucose oxidase, $200 \mathrm{mg} / \mathrm{ml}$ ammonium chloride, and $250 \mathrm{mg} / \mathrm{ml} \mathrm{D}-(+)$-glucose. Sections were reacted until optimal staining intensity was achieved (sections from injured animals and sham controls were processed together in the same wells) and then washed in Tris, placed on slides, dried, dehydrated in graded ethanol and xylene, and coverslipped with Permount. Immunocytochemical control sections received the identical treatment except for exposure to the primary antibody, and showed no specific staining.

Hippocampal cell counts. For cell counts of hilar neurons, six nonadjacent, $30-\mu \mathrm{m}$-thick coronal sections from the dorsal hippocampus (three from bregma -3.3 and three from bregma -4.2 ) stained with toluidine blue were analyzed in a blinded fashion for cach animal. Hilar neurons were considered as those cells lying within the region defined by the superior and inferior dentate granule cell layers, excluding the $\mathrm{CA} 3 \mathrm{c}$ pyramidal neurons and transitional neurons within approximately $20 \mu \mathrm{m}$ of the pyramidal neurons. Neurons were counted only if there was a clearly defined cell body and nucleus. For cell counts of other neuronal subpopulations, $20 \mu \mathrm{m}$ sections were used to quantify densely packed cell layers. These regions included the CA3c pyramidal neurons, the superior dentate granule cell layer beginning directly above the medial extent of $\mathrm{CA} 3 \mathrm{c}$ and extending $200 \mu \mathrm{m}$ laterally, and CA 1 pyramidal neurons located directly above the lateral end of the superior dentate granule cell layer and extending $200 \mu \mathrm{m}$ laterally. In the main experiments, values from controls were compared to each injury group using a one-way analysis of variance (ANOVA). When significant differences were found, individual groups were compared using Student's $t$ test with the Bonferroni correction for multiple comparisons. In the experiments using isoflurane anesthesia, cell counts from the injured and control groups were compared using the unpaired Student's $t$ test.

Electrophysiology. Animals were anesthetized with subcutaneous urethane $(1.25 \mathrm{gm} / \mathrm{kg})$ I week after trauma or sham surgery and placed in a stereotactic frame. Bipolar stimulating electrodes (SNE-200, Rhodes Medical Instruments) were lowered into the perforant path bilaterally, and glass electrodes filled with $4 \mathrm{M} \mathrm{NaCl}$ were inserted into the dentate granule cell layer bilaterally using methods described previously (Sloviter, 1983; Lowenstein et al., 1991a). Perforant path stimuli were delivered by a Grass S-88 stimulator connected to a stimulus isolation unit. Evoked responses were amplified and displayed on a digital oscilloscope. The stimulus voltage was first adjusted to obtain maximal dentate granulc ccll population spikes, and baseline extracellular responses to 0.2 $\mathrm{Hz}$ paired-pulse stimuli (duration, $0.1 \mathrm{msec}$; interstimulus interval, 20 , 40,60 , or $80 \mathrm{msec}$ ) were recorded. Similar measurements were then made using stimulus frequencies of $1.0,2.0,3.0,4.0$, and $5.0 \mathrm{~Hz}$, with approximately 2 min intervals between changes in frequency. The threshold for frequency-dependent loss of dentate granule cell inhibition was defined as the stimulus frequency that led to multiple population spikes and afterdischarges. Under normal circumstances, this usually appeared 15-20 sec after the frequency had been increased to $3 \mathrm{~Hz}$ or higher. All observations were made while blinded to the prior treatment.

\section{Results}

Behavior of animals after impact. Following fluid-percussion injury, all animals were observed continuously until fully recovered from anesthesia. Physiological monitoring of blood pressures and arterial blood gases during the first 15 min after impact showed no evidence of significant cardiorespiratory compromise in the animals (Table 1). Animals receiving mild (0.5-1.2 atm) and moderate (1.5-1.6 atm or $1.8 \mathrm{~atm})$ impacts recovered fully from the anesthesia within $20 \mathrm{~min}$ of the impact, and their subsequent behavior such as feeding and grooming were normal. The return to normal activity after anesthesia was delayed $2-3 \mathrm{hr}$ in animals that received impacts of $2.0 \mathrm{~atm}$ or higher. Frequent daily monitoring following recovery showed no evidence of abnormal behavior or clinical seizure activity in any of the experimental groups. The weights of animals before impact and $7 \mathrm{~d}$ later were not significantly different between the controls and three treatment groups.

Effects of impacts on gross brain structure and cortical histology. Brains from animals $(n=12)$ killed 1 week after mild impact had no obvious external injury and no gross distortion of the hippocampi. The cerebral cortex immediately under the impact site had some loss of neurons, but the surrounding cortex was normal. Brains from animals that received the moderate ( $n$ $=8)$ and high impacts $(n=7)$ had cavitation of the cortex at the impact site and some medial distortion of pyramidal cell layers adjacent to the lateral ventricle. Some animals in these groups also had small hemorrhages in the periventricular white matter immediately below the injured cortex. None of the animals had gross evidence of injury to the contralateral hemisphere. 

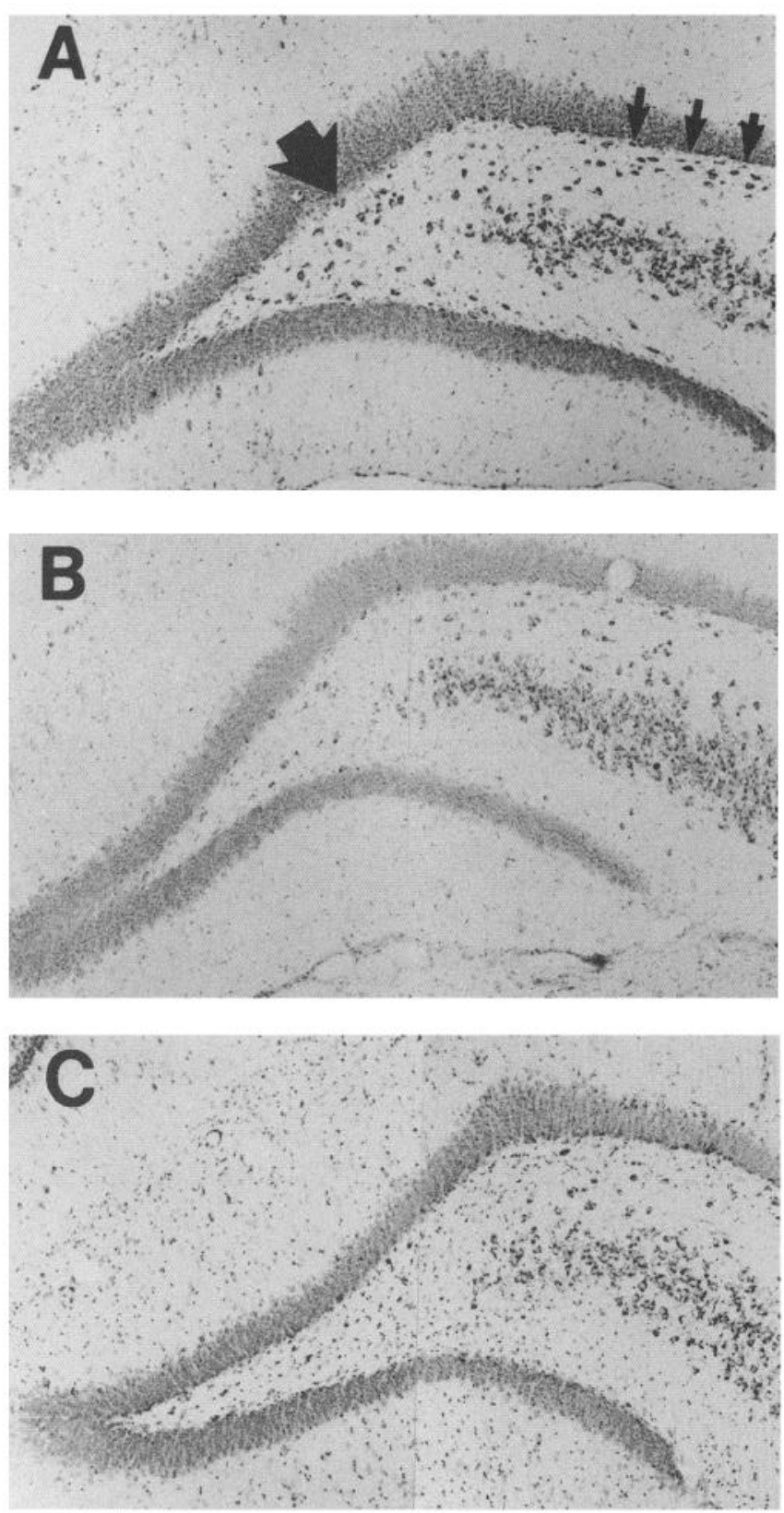

Figure 1. Histological evidence of hilar neuron injury following traumatic brain injury: photomicrographs of $30 \mu \mathrm{m}$ toluidine blue-stained tissue sections from the ipsilateral, dorsal hippocampus 1 week following sham treatment $(A), 1.1 \mathrm{~atm}$ injury $(B)$, and $2.2 \mathrm{~atm}$ injury $(C)$. Note the substantial number of large neuronal cells within the deep hilus (large arrow) and immediately below the superior dentate granule cell layer (small arrows) in the control, and the obvious decrease in identifiable neurons following injury. The smaller, basophilic elements in the $2.2 \mathrm{~atm}$ injury hilus are consistent with gliosis; this was verified with immunocytochemistry for glial fibrillary acidic protein, a specific marker of astrocytes, on adjacent sections (data not shown). Magnification, $92 \times$.

Effects of impacts on hippocampal structure. Examination of hippocampal sections 1 week after trauma revealed a significant reduction in the number of dentate hilar neurons on the side ipsilateral to the impact in all three experimental groups (Figs. $1,2)$. There was a less extensive but clear decrease in neurons of the contralateral hilus as well (Fig. 2). The degree of hilar neuron loss increased in both hippocampi as the impact force increased. The neuronal cell loss appeared highly selective since the overall number and morphology of neighboring dentate granule and pyramidal cells appeared normal in all groups. This was verified quantitatively by cell counts in representative regions of the dentate granule cell layer, and CA3c and CA1 pyramidal cell layers in five animals that received the highest impact and in five sham controls. No differences were observed in any of these regions on either side of the brain (Fig. 2). However, a small area of the pyramidal cell layer (CA3/CA2 in the dorsal hippocampus and CA1 in the ventral hippocampus) was injured following the higher impact forces. The location of injured pyramidal neurons was consistently adjacent to the lateral ventricle and in line with the direction of the fluid-percussion impact.

Immunocytochemical staining showed a substantial reduction in somatostatin-immunoreactive hilar neurons 1 week after injury (Fig. $3 A, B$ ). Evidence for hilar injury was also found in hippocampal sections from animals killed $4 \mathrm{hr}$ after trauma. Some neurons had basophilic, cytoplasmic aggregates similar to those seen in other acute injury states (Brown and Brierley, 1968), and neurons became impregnated by a silver degeneration stain (Fig. 3C-E). The morphology of many of the degenerating neurons was consistent with mossy cells, which are thought to comprise the majority of hilar neurons (Amaral, 1978).

Comparison of different anesthetic agents on trauma-induced hippocampal injury. To assess whether the trauma-induced pattern of hippocampal injury was specific to a particular anesthetic agent, a group of animals was anesthetized with isoflurane (instead of pentobarbital) and subjected to either an intermediate level of impact (1.8 atm; $n=3)$ or sham surgery $(n=3)$. Physiologic monitoring again showed stable systemic blood pressures and arterial blood gas measurements following the impact (Table 1). The appearance of the brains from these animals 1 week after impact looked very similar to those from the mild impact group anesthetized with pentobarbital. That is, there was no apparent external injury, low-power views of sections showed no distortion of the hippocampi, and closer inspection of the cortex revealed some loss of neurons immediately below the impact site. The animals anesthetized with isoflurane had a pattern of selective loss of hilar neurons that was identical to the pattern observed following pentobarbital anesthesia. Cell counts revealed a significant loss of hilar neurons bilaterally that was most prominent ipsilateral to the impact (Fig. 4). Furthermore, cell counts of adjacent hippocampal regions confirmed that the hilar cell loss was highly selective, similar to the observations made following pentobarbital anesthesia (Fig. 2).

Effects of impacts on dentate granule cell physiology. A number of recent studies suggest that hilar neurons have an important role in governing the excitability of dentate granule cells (Scharfman et al., 1990; Scharfman, 1991; Sloviter, 1991). We therefore examined the potential functional consequences of trauma-induced hilar neuron loss on dentate gyrus physiology. Extracellular recordings of dentate granule cell layer field potentials evoked by perforant path stimulation were measured in vivo 1 week following brain injury. Paired-pulse recordings were done blinded on a subset of the same injured $(n=21)$ or control $(n=7)$ animals used for the histologic analyses described above. (These animals were anesthetized with pentobarbital during trauma.) Dentate granule cell responses during 0.2, 1.0, and 2.0 $\mathrm{Hz}$ stimulation were normal bilaterally in all control animals and some animals following mild or moderate injury (Fig. $5 A$ ). In contrast, most animals with moderate injury had a frequencydependent loss of inhibition in the ipsilateral hippocampus, as 
Table 1. Acute physiologic measurements during traumatic brain injury

\begin{tabular}{|c|c|c|c|c|}
\hline & 5 min preinjury & $5 \mathrm{~min}$ post & $10 \mathrm{~min}$ post & 15 min post \\
\hline \multicolumn{5}{|l|}{ Pentobarbital anesthesia } \\
\hline Systolic BP (mm Hg) & $129 \pm 18$ & $131 \pm 30$ & $129 \pm 24$ & - \\
\hline pH & $7.40 \pm 0.05$ & $7.40 \pm 0.04$ & $7.30 \pm 0.10$ & $7.32 \pm 0.03$ \\
\hline $\mathrm{pO}_{2}(\mathrm{~mm} \mathrm{Hg})$ & $71.5 \pm 12.4$ & $73.5 \pm 12.6$ & $71.8 \pm 13.8$ & $77.8 \pm 11.3$ \\
\hline $\mathrm{pCO}_{2}(\mathrm{~mm} \mathrm{Hg})$ & $44.6 \pm 5.2$ & $43.0 \pm 4.2$ & $41.9 \pm 7.5$ & $40.6 \pm 13.6$ \\
\hline \multicolumn{5}{|l|}{ Isoflurane anesthesia } \\
\hline Systolic BP (mm Hg) & $128 \pm 11$ & $134 \pm 11$ & $129 \pm 10$ & $127 \pm 9$ \\
\hline $\mathrm{pH}$ & $7.40 \pm 0.04$ & $7.39 \pm 0.04$ & $7.39 \pm 0.03$ & $7.40 \pm 0.03$ \\
\hline $\mathrm{pO}_{2}(\mathrm{~mm} \mathrm{Hg})$ & $92.8 \pm 6.0$ & $92.4 \pm 5.6$ & $92.2 \pm 6.9$ & $94.0 \pm 6.3$ \\
\hline $\mathrm{pCO}_{2}(\mathrm{~mm} \mathrm{Hg})$ & $39.0 \pm 2.7$ & $39.1 \pm 2.2$ & $39.4 \pm 1.5$ & $39.3 \pm 1.4$ \\
\hline
\end{tabular}

Measurements of systolic blood pressure (BP) and arterial blood gases in animals anesthetized with either pentobarbital (2.0-2.2 atm impact, $n=4$ ) or isoflurane (1.8 atm impact, $n=3$ ) are shown. Values are mean $\pm \mathrm{SD}$; none of the measurements during the postinjury period were significantly different from preinjury control values.

evidenced by multiple population spikes elicited by stimulation frequencies of $2.0 \mathrm{~Hz}$ or less (Fig. $5 A$ ). Furthermore, animals that received the highest impacts had bilateral abnormalities in hippocampal electrophysiology. These included bilateral frequency-dependent disinhibition at $2.0 \mathrm{~Hz}$ or less, or extremely low-amplitude field potentials ipsilateral to the injury (likely due to effects of the trauma on the perforant path) and contralateral disinhibition. There was a strong correlation between the abnormal granule cell excitability, number of hilar neurons, and the severity of injury (Fig. $5 B$ ).

\section{Discussion}

The main finding of this study is that a single, brief impact applied to the surface of the cerebral cortex causes a selective, bilateral loss of hilar neurons and persistent changes in dentate granule cell excitability. A direct relationship between the trau- matic impact and hippocampal injury was supported by the observation that increasing forces of impact led to a greater amount of hilar neuron loss. The neuronal loss documented by cell counts was further substantiated by the identification of degenerating hilar neurons a few hours after impact, and a decrease in somatostatin-immunoreactive cells in the hilus 1 week later. Furthermore, selective hilar neuron loss was observed following experiments using two different anesthetic agents, suggesting that this phenomenon is not restricted to the pharmacologic conditions created by a particular anesthetic.

This pattern of trauma-induced hilar neuron loss shares features with the "selective vulnerability" of hippocampal neurons following other forms of CNS injury such as ischemia (Smith et al., 1984; Johansen et al., 1987) and prolonged seizure activity (Sloviter, 1987). However, there are a number of reasons to suggest that trauma-induced hilar injury is a unique process that
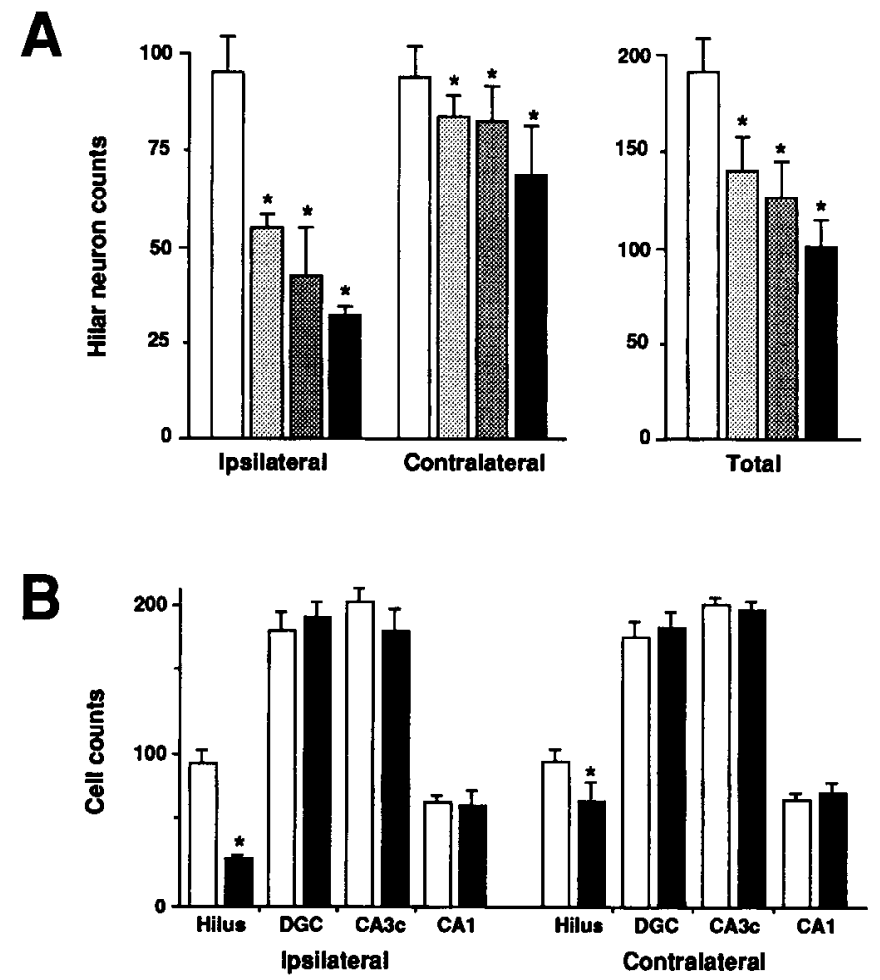



Figure 2. Hippocampal neuron counts 1 week following traumatic brain injury. $A$, The number of hilar neurons (ipsilateral to impact, contralateral to impact, and total for both hippocampi) in the three injury groups are compared to controls. Valucs shown are mean \pm SD. All three comparisons are significant at $p<0.0001$ (ANOVA); ${ }^{*}, p<$ 0.05 by post hoc Student's $t$ test with the Bonferroni correction for multiple comparisons. $B$, Neuron counts in the hilus, dentate granule cell layer $(D G C)$, and $C A 3 \mathrm{C}$ and $\mathrm{CAl}$ pyramidal cell layer (see Materials and Methods for details) are compared between the highest impact group (2.0-2.2 atm) and controls. $*, p<0.05$ by Student's $t$ test. Note the lack of significant differences in regions other than the hilus. 



Figure 3. Somatostatin immunocytochemistry of the dorsal hippocampus 1 week following sham treatment $(A)$ and a 2.2 atm injury $(B)$. Note the almost complete loss of somatostatin-immunoreactive neurons in the ipsilateral deep hilus following injury. $C$, Toluidine blue staining of two neurons (arrows) from the ipsilateral, deep hilus $4 \mathrm{hr}$ after a $1.1 \mathrm{~atm}$ injury shows shrinkage of the cytoplasmic boundaries and basophilic stippling consistent with early cellular injury; another neuron directly between the injured neurons appears normal. $D$, Silver degeneration staining 4 hr after a $1.5 \mathrm{~atm}$ injury shows numerous positively stained hilar neurons; no neuronal staining was observed in control animals $(E)$. Magnification: $A$ and $B, 110 \times ; C, 365 \times ; D$ and $E, 194 \times$.

is not explained by these previously known pathogenetic mechanisms. First, there was no physiologic evidence of global ischemia or hypoxia following the highest impact forces, since systemic blood pressure and oxygenation remained normal during the acute period after trauma. Substantial global ischemia or hypoxia seems especially unlikely in the animals receiving the mild and moderate impacts, since they recovered fully from anesthesia within $20 \mathrm{~min}$ of the injury. Second, significant seizure-induced injury was unlikely since most of the experiments were done with pentobarbital anesthesia, no clinical seizures were observed following recovery from anesthesia, and no repetitive hippocampal seizures were seen during the recordings made 1 week after injury. Finally, the hippocampal injury after trauma was unique since it remained relatively confined to the hilus even after the most severe impacts. This is in contrast, for example, to ischemic injury in which it is common to see a combination of hilar and CA1 pyramidal neuron loss after relatively mild ischemia (Pulsinelli et al., 1982; Smith et al., 1984; Simon et al., 1991).

These observations have implications regarding our under-
Figure 4. Hippocampal neuron counts 1 week following traumatic brain injury in animals anesthetized with isoflurane during impact. Neuron counts in the hilus, dentate granule cell layer $(D G C)$, and $C A 3 \mathrm{c}$ and $\mathrm{CA} 1$ pyramidal cell layer are compared between animals exposed to a $1.8 \mathrm{~atm}$ impact and sham-operated controls. ${ }^{*}, p<0.001 ;{ }^{* *}, p<0.03$ by Student's $t$ test. Note the lack of significant differences in regions other than the hilus.

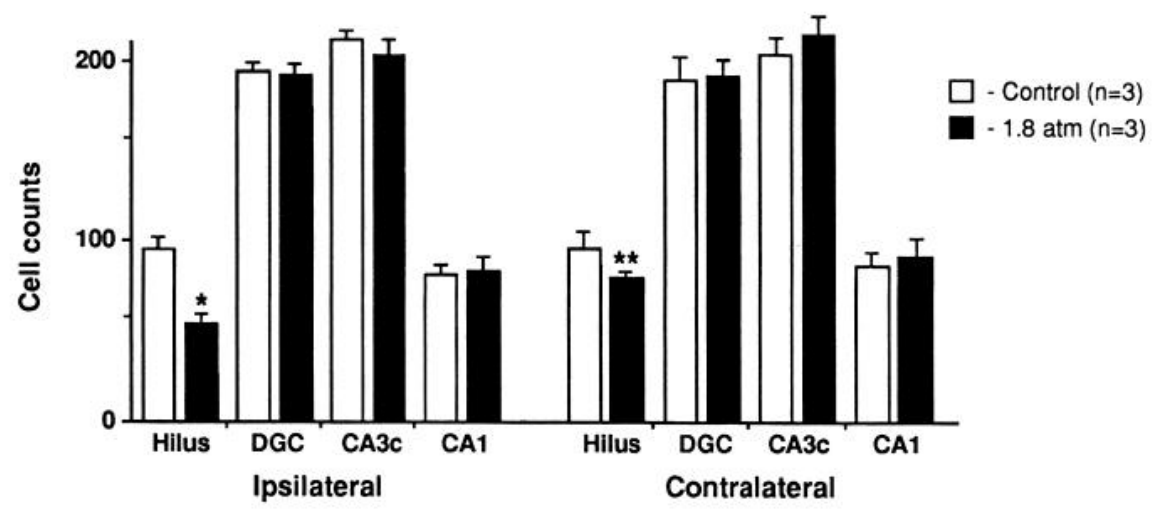


B

Control

$1.0 \mathrm{~Hz}$

$2.0 \mathrm{~Hz}$

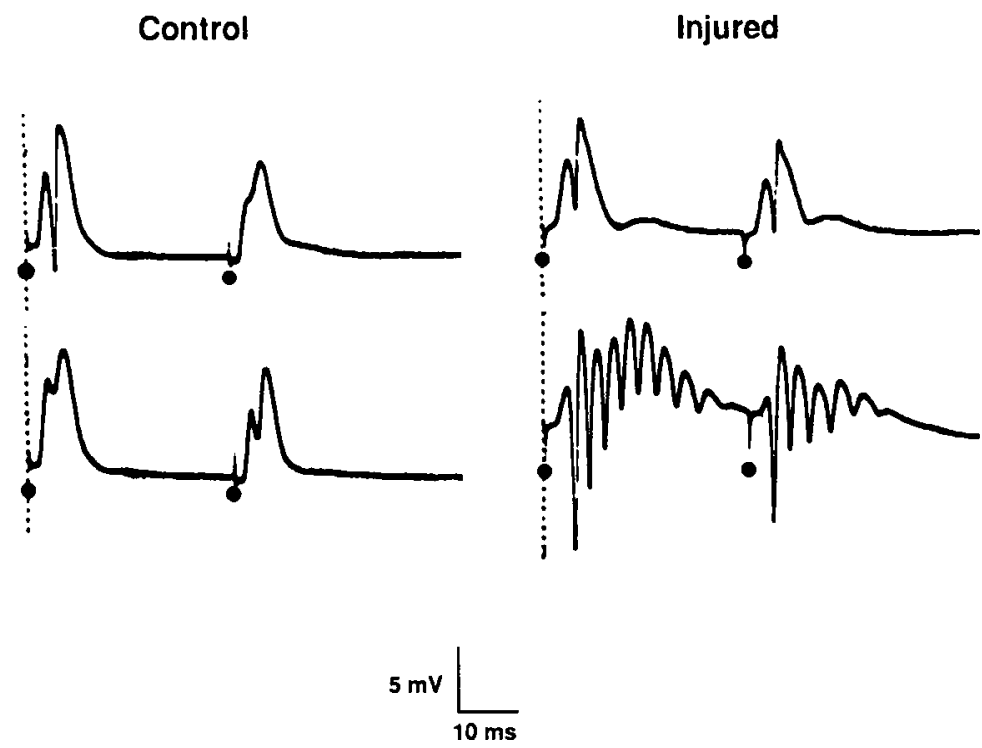

Injured
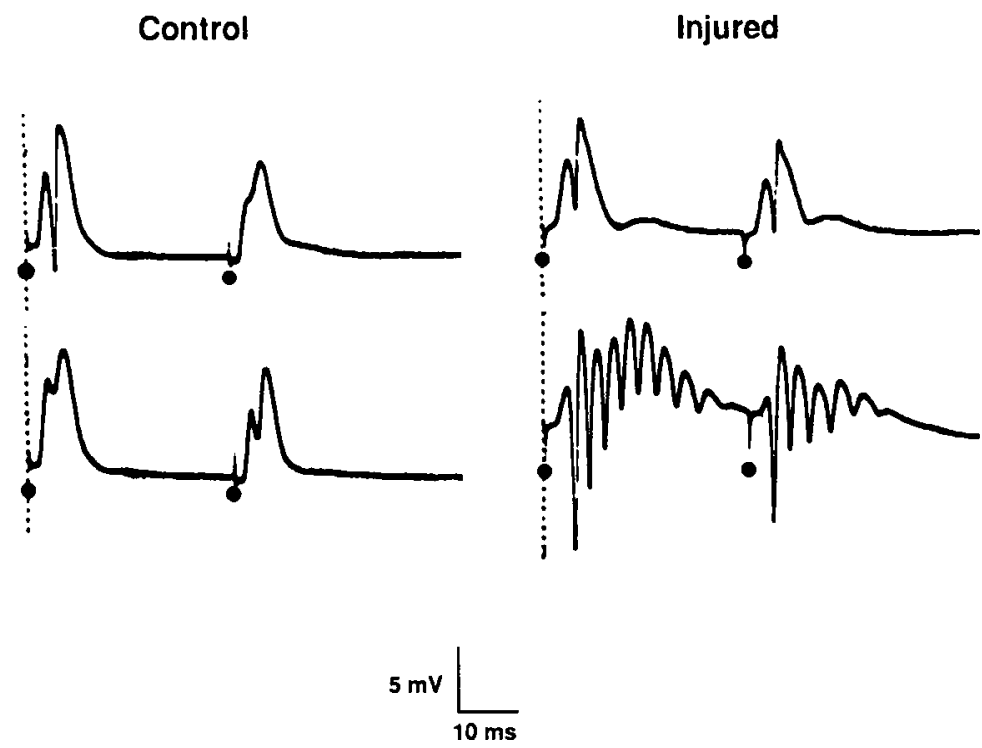

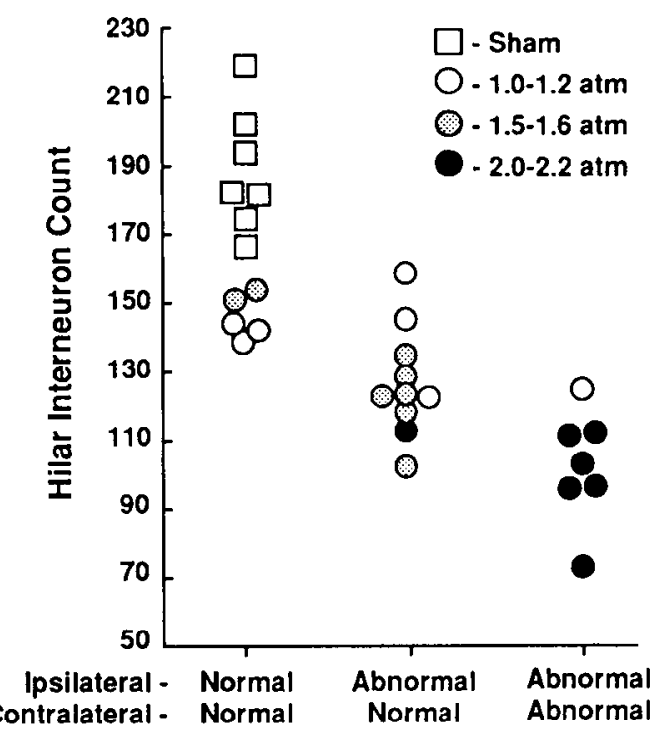

Response to $2.0 \mathrm{~Hz}$ Stimulation

Figure 5. Hippocampal electrophysiology following traumatic brain injury. A, Responses in a control (left) and 1.5 atm injury (right) animal to paired pulses with a $40 \mathrm{msec}$ interval at 1.0 and $2.0 \mathrm{~Hz}$. Note the loss of inhibition at $2.0 \mathrm{~Hz}$ in the injured hippocampus as evidenced by the appearance of large, multiple dentate granule population spikes. This was more typically seen with an 80 msec interstimulus interval but in some animals was present with a $40 \mathrm{msec}$ interval as shown. Solid circles indicate the stimulus artifact. $B$, The relationship of the electrophysiology to hilar neuron count and injury severity is shown graphically. The electrophysiology for each animal was categorized as either normal bilaterally, abnormal ipsilateral to injury, or abnormal bilaterally (the latter included $2.0 \mathrm{~Hz}$ disinhibition bilaterally, or low-amplitude recordings ipsilateral and $2.0 \mathrm{~Hz}$ disinhibition contralateral to injury).

standing of the pathogenesis of temporal lobe epilepsy. The connection between head trauma and epilepsy of various kinds has been recognized since antiquity (Jennett, 1975). Although temporal lobe epilepsy may have multiple causes, in some patients the only identifiable etiologic event is a history of closed head trauma (Hauser and Kurland, 1975; Bruton, 1988). Pathological studies of these patients have revealed a spectrum of findings, ranging from cortical and white matter scars to isolated "Ammon's horn sclerosis," in which there is a selective loss of dentate hilar neurons and variable involvement of the pyramidal cell layers (Margerison and Corsellis, 1966; Bruton, 1988). In some patients, the damage is restricted entirely to the hilus ("end-folium sclerosis"), and this pattern is virtually identical to what we have observed in the present study. The patterns are also similar in that a loss of somatostatin-immunoreactive hilar neurons has been observed in these patients (Robbins et al., 1991). Interestingly, the hilar cell loss that we found may also share some features with the hippocampal injury described in boxers with dementia pugilistica (Corsellis et al., 1973). Thus, our observations are consistent with a clinical precedent that suggests an association between single or repeated episodes of head trauma and a selective loss of hilar neurons.

The physiological relevance of hilar cell loss to seizures and epilepsy has been explored in various experimental studies. For example, Sloviter $(1987,1991)$ has shown that prolonged stimulation of the perforant path destroys hilar neurons ipsilateral to the side of stimulation, including the somatostatin-containing neurons as seen in the present study. Electrophysiologic recordings in these perforant path animals show relative hyperexcitability of dentate granule cells that are similar to our observations. Analogous changes in granule cell excitability have been seen in acute recordings from hippocampi removed for the treatment of temporal lobe epilepsy (Williamson et al., 1990). Thus, our observations lend further support to the concept that hilar neurons are an important component of the normal local inhibitory circuitry that controls dentate granule cell excitability. More importantly, the relationships between trauma, hilar cell loss, and dentate granule cell hyperexcitability shown here are consistent with the emerging hypothesis that dysfunction or loss of hilar neurons may explain the pathogenesis of certain forms of temporal lobe epilepsy (Sloviter, 1991).

Another principal observation in this study is that a single, unilateral impact can lead to a bilateral loss of hippocampal neurons and bilateral abnormalities in hippocampal physiology. A variety of hippocampal lesions have been shown to result in memory deficits in humans and experimental animals, but the lesions have consistently involved both hippocampi (Scoville and Milner, 1957; Kesner and Novak, 1982; Zola-Morgan et al., 1986). It remains to be determined whether animals receiving impact forces of 1.0-2.2 atm, especially those that have apparent bilateral hippocampal injury, develop an impairment in memory. However, previous studies using the same model of traumatic injury have demonstrated that animals injured with higher impact forces (i.e., $2.6 \mathrm{~atm}$ ) have a disturbance of visualspatial memory as measured in the Morris water maze (Smith et al., 1991). Lyeth et al. (1990) have also shown that a model of midline percussion injury in the rat causes a visual-spatial learning deficit. These latter investigators did not observe any obvious hippocampal injury, although the focus of their study was on CAl pyramidal neurons rather than the hilus. The findings reported in the present study provide clear evidence that bilateral hippocampal dysfunction may arise from traumatic 
brain injury, and they provide a potential anatomic basis for this impairment. Furthermore, the spectrum of neuronal injury we observed over a range of impact forces raises the possibility that even milder impacts may produce ipsilateral hilar neuron loss and contralateral hilar neuron dysfunction, or bilateral dysfunction without actual cell loss. This may be one mechanism whereby mild head injury causes transient memory loss that then returns to normal. Future studies that correlate the permanence of memory loss with the pattern and degree of cell injury in this model should clarify this issue.

Although the clinical situations modeled in this study cannot be defined precisely, some estimate is possible based on the gross pathological changes produced by the fluid-percussion technique. The lowest impact forces caused minimal cortical scarring, and this would parallel changes that have been documented after mild closed head injury in humans (Graham et al., 1989). In contrast, animals receiving the highest impact forces developed cavitation of the underlying cortex and adjacent white matter. This is similar to the more severe blunt head injury in humans that causes a depressed skull fracture and more extensive cortical contusion and hemorrhage. It is unknown whether the hilar injury observed in this study could result from forces applied to the external surface of the human skull. Howcver, the human temporal lobes are especially susceptible to forces generated by head trauma (Gurdjian and Gurdjian, 1976). This is primarily due to their anatomic relationship to the angular contours of the floor of the orbital fossa and the sphenoid wing. These observations make it quite likely that the human hippocampus can be exposed to mechanical forces that are similar to the conditions that produced hilar injury in the present study.

The mechanisms underlying trauma-induced hilar injury remain unknown. Our working hypothesis is that the cell loss is directly related to the mechanical effects of the impact on the hippocampus. This is consistent with the very rapid onset of injury. Mechanical effects could also explain the presence of bilateral hilar injury, since the percussive wave is likely to cross the midline into the contralateral hemisphere. It is also possible that other significant pathogenetic mechanisms, such as focal ischemia restricted to the hilus, are superimposed upon the mechanical effects of the impact. Nonetheless, it remains unclear exactly how a mechanical force would cause such highly selective injury to such a relatively restricted part of the brain. It is conceivable that the particular location and structural configuration of the hilus make it especially susceptible to mechanical forces. However, given the vulnerability of hilar neurons to other nonmechanical forms of injury, it seems likely that intrinsic cellular factors explain their sensitivity to trauma. Such intrinsic factors may include the ability of the cells to maintain calcium homeostasis via calcium binding proteins (Scharfman and Schwartzkroin, 1989), or the repertoire of stress proteins induced by injury (Lowenstein et al., 1991b). The characterization of the factors that determine these pathophysiological events may ultimately lead to more specific treatment for head-injured patients.

Note added in proof. A recent study by Gallyas and Zoltay (Acta Neuropathol 83:386-393, 1992) provides evidence for direct mechanical injury of neurons distant from the impact site within 1 minute of concussive head trauma in the rat.

\section{References}

Adams J, Graham D, Murray L, Scott G (1982) Diffuse axonal injury due to non-missile head injury in humans: an analysis of 45 cases. Ann Neurol 12:557-563.

Amaral D (1978) A Golgi study of cell types in the hilar region of the hippocampus in the rat. J Comp Neurol 182:851-914.

Amaral D (1987) Memory: Anatomical organization of candidate brain regions. In: Handbook of physiology: the nervous system. Vol. V. Higher functions of the brain, part I (Mountcastle V, Plum F, Geiger S, eds), pp 211-294. Bethesda, MD: American Physiology Society.

Binder L (1986) Persistent symptoms after mild head injury: a review of the postconcussive syndrome. J Clin Exp Neuropsychol 8:323346.

Brown A, Brierley J (1968) The nature, distribution and earliest stages of anoxic-ischemic nerve cell damage in rat brain. Br J Exp Pathol 44:87-106

Bruton C (1988) The neuropathology of temporal lobe epilepsy. New York: Oxford UP.

Corsellis J, Bruton C, Freeman-Browne D (1973) The aftermath of boxing. Psychol Med 3:270-303.

Dewitt D, Jenkins L, Wei E, Lutz H, Becker D, Kontos H (1986) Effects of fluid-percussion on regional cerebral blood flow and pial vessel diameter. J Neurosurg 64:787-794.

Engel J (1989) Seizures and epilepsy. Contemporary neurology series. Philadelphia: FA Davis.

Gentilini M, Nichelli P, Schoenhuber R, Bortolotti P, Tonelli L, Falasca A, Merli G (1985) Neuropsychological evaluation of mild head injury. J Neurol Neurosurg Psychiatry 48:137-140.

Graham DI, Lawrence AE, Adams JH, Doyle D, McLellan D, Gennarelli TA (1989) Pathology of mild head injury. In: Mild to moderate head injury (Hoff J, Anderson T, Cole T, eds), pp 63-75. Boston: Blackwell Scientific.

Gurdjian ES, Gurdjian ES (1976) Cerebral contusions: re-evaluation of the mechanism of their development. J Trauma 16:35-51.

Hauser WA, Kurland LT (1975) The epidemiology of epilepsy in Rochester, Minnesota, 1935 through 1967. Epilepsia 16:1-66.

Hayes R, Lyeth B, Jenkins L (1989) Neurochemical mechanisms of mild and moderate head injury; implications for treatment. In: Mild head injury (Levin H, Eisenberg H, Benton A, eds), pp 54-79. New York: Oxford UP.

Jennett B (1975) Epilepsy after non-missile head injuries. London: Heinemann.

Johansen F, Zimmer J, Diemer N (1987) Early loss of somatostatin neurons in dentate hilus after cerebral ischemia in the rat precedes CA-1 pyramidal cell loss. Acta Neuropathol (Berl) 73:110-114.

Kesner R, Novak J (1982) Serial position curve in rats: role of the dorsal hippocampus. Science 218:173-175.

Kraus J (1987) Epidemiology of head injury. In: Head injury (Cooper $P$, ed). Baltimore: Williams and Wilkins.

Levin H (1989) Neurobehavioral outcome of mild to moderate head injury. In: Mild to moderate head injury (Hoff J, Anderson T, Cole T, eds), pp 153-185. Boston: Blackwell Scientific.

Levin H, Mattis S, Ruff R, Eisenberg H, Marshall L, Tabaddor K, High W, Frankowski R (1987) Neurobehavioral outcome following minor head injury: a three-center study. J Neurosurg 66:234-243.

Lewelt W, Jenkins L, Miller J (1980) Autoregulation of cerebral blood flow after experimental fluid percussion injury of the brain. J Neurosurg 53:500-511.

Lowenstein D, Miles M, Hatam F, McCabe T (1991a) Up-regulation of calbindin-D28K mRNA in the rat hippocampus following focal stimulation of the perforant path. Neuron 6:627-633.

Lowenstein D, Chan P, Miles M (1991b) The stress protein response in cultured neurons: characterization and evidence for a protective role in excitotoxicity. Neuron 7:1053-1060.

Lyeth B, Jenkins L, Dixon R, Dixon C, Phillips L, Clifton G, Young H, Hayes R (1990) Prolonged memory impairment in the absence of hippocampal cell death following traumatic brain injury in the rat. Brain Res 526:249-258.

Margerison J, Corsellis J (1966) Epilepsy and the temporal lobes. Brain 89:499-530.

McIntosh T, Vink R, Noble L, Yamakami S, Soares H, Faden A (1989) Traumatic brain injury in the rat: characterization of a lateral fluidpercussion model. Neuroscience 28:233-244.

Nadler J, Evenson D (1983) Use of excitatory amino acids to make 
axon-sparing lesions of hypothalamus. Methods Enzymol 103:393400.

Oppenheimer D (1968) Microscopic lesions in the brain following head injury. J Neurol Neurosurg Psychiatry 31:299-306.

Pilz P (1983) Axonal injury in head injury. Acta Neurochir [Suppl] (Wien) 32:119-123.

Povlishock J, Becker D (1985) Central nervous system trauma status report. Washington: Byrd.

Povlishock J, Becker D, Cheng C, Vaughan G (1983) Axonal change in minor head injury. $J$ Neuropathol Exp Neurol 42:225-242.

Pulsinelli W, Brierley J, Plum F (1982) Temporal profile of neuronal damage in a model of transient forebrain ischemia. Ann Neurol 11: $491-498$.

Rimel R, Giordani B, Barth J, Boll T, Jane J (1981) Disability caused by minor head injury. Neurosurgery 9:221-228.

Robbins R, Brines M, Kim J, Adrian T, de Lanerolle N, Welsh S, Spencer D (1991) A selective loss of somatostatin in the hippocampus of patients with temporal lobe epilepsy. Ann Neurol 29:325-332.

Sagar S, Marshall P, Landis D (1985) Immunoreactive somatostatin in the rat retina: light microscopic immunocytochemistry and chromatographic characterization. Brain Res 336:235-242.

Scharfman H (1991) Dentate hilar cells with dendrites in the molecular layer have lower thresholds for synaptic activation by perforant path than granule cells. J Neuroscience 11:1660-1673.

Scharfman H, Schwartzkroin P (1989) Protection of dentate hilar cells from prolonged stimulation by intracellular calcium chelation. Science 246:257-260.

Scharfman H, Kunkel D, Schwartzkroin P (1990) Synaptic connections of dentate granule cells and hilar neurons: results of paired intracellular recordings and intracellular horseradish peroxidase injections. Neuroscience 37:693-707.

Scoville W, Milner B (1957) Loss of recent memory after bilateral hippocampal lesions. J Neurol Neurosurg Psychiatry 20:11-21.

Simon R, Cho H, Gwinn R, Lowenstein D (1991) The temporal profile of 72-kDa heat-shock protein expression following global ischemia. J Neurosci 11:881-889.
Sloviter R (1983) "Epileptic" brain damage in rats induced by sustained electrical stimulation of the perforant path. I. Acute electrophysiological and light microscopic studies. Brain Res Bull 10:675697.

Sloviter R (1987) Decreased hippocampal inhibition and a selective loss of interneurons in experimental epilepsy. Science 235:73-76.

Sloviter R (1991) Permanently altered hippocampal structure, excitability, and inhibition after experimental status epilepticus in the rat: the "dormant basket cell" hypothesis and its possible relevance to temporal lobe epilepsy. Hippocampus 1:41-66.

Sloviter R, Damiano B (1981) Sustained electrical stimulation of the perforant path duplicates kainate-induced electrophysiological effects and hippocampal damage in rats. Neurosci Lett 24:279-284.

Smith D, Okiyama K, Thomas M, Claussen B, McIntosh T (1991) Evaluation of memory dysfunction following experimental brain injury using the Morris water maze. J Neurotrauma 8:259-269.

Smith M, Auer R, Siesji B (1984) The density and distribution of ischemic injury in the rat following 2-10 minutes of forcbrain ischemia. Acta Neuropathol (Berl) 64:319-332.

Squire L, Zola-Morgan S (1991) The medial temporal lobe memory system. Science 253:1380-1386.

Strauss I, Savitsky N (1934) Head injury: neurologic and psychiatric aspects. Arch Neurol Psychiatry 31:893-955.

Strich S (1956) Diffuse degeneration of the cerebral white matter in severe dementia following head injury. J Neurol Neurosurg Psychiatry 19:163-185.

Williamson A, McCormick D, Shepherd C, Spencer D (1990) Intracellular recordings from epileptic human dentate granule cells show evidence of hyperexcitability. Epilepsia 31:625.

Yamakami S, McIntosh T (1989) Effects of traumatic brain injury on regional cerebral blood flow in rats as measured with radiolabeled microspheres. J Cereb Blood Flow Metab 9:117-124.

Zola-Morgan S, Squire L, Amaral D (1986) Human amnesia and the medial temporal region: enduring memory impairment following a bilateral lesion limited to field $\mathrm{CA} 1$ of the hippocampus. J Neurosci 6:2950-2967. 